Elsevier Editorial System(tm) for Fusion Engineering and Design

Manuscript Draft

Manuscript Number: FUSENGDES-D-10-00164

Title: Dynamic response analysis of superconducting coils in Wendelstein 7-X and mechanical quench test

Article Type: FUSION TECHNOLOGY 2010 SI

Keywords: Superconducting coil, mechanical disturbance, mechanical quench, dynamic response, FEM, W7-X

Corresponding Author: Dr. Joris Fellinger, Ph.D.

Corresponding Author's Institution: Max-Planck-Institut für Plasmaphysik

First Author: Joris Fellinger, Dr.

Order of Authors: Joris Fellinger, Dr.; Stefan Freundt; Dag Hathiramani, Dr.; Victor Bykov; Felix

Schauer, Dr.

Abstract: The Wendelstein 7-X (W7-X) modular stellarator is under construction at the Max-PlanckInstitut für Plasmaphysik in Greifswald, Germany. The W7 X magnet system contains 70

superconducting (sc) coils which are supported by a massive central steel ring structure via bolted connections and by numerous welded as well as gliding inter-coil elements.

Load tests on prototypes of bolted coil to support ring connections whose flanges partially open during high field operation, and of sliding support structures between the coils showed that stick-slip events at their contact surfaces cause shock waves. In order to assess the risk of a shock-induced quench of the superconductor, one of the coils was subjected to impact loads - the so called mechanical quench (MQ) test - in the course of the cryogenic acceptance tests at CEA in Saclay, France. No quench could be triggered even with the lowest coil stability margin.

The paper demonstrates that the amplitudes of the shock waves in the W7-X magnet system are comparable to those generated by the impacts in the MQ tests. For that purpose, finite element (FE) models of both W7-X and the MQ test simulating the dynamic responses were developed and successfully validated against measurements. 
Greifswald, 30.08.2010

Dear Sir, Dear Madam,

Please find attached the paper for the SOFT 2010 entitled:

"Dynamic response analysis of superconducting coils in Wendelstein 7-X and mechanical quench test"

Coauthors:

Stefan Freundt, Dag Hathiramani, Victor Bykov, Felix Schauer

Thank you very much for considering our paper.

Yours sincerely,

Joris Fellinger

Max-Planck-Institut für Plasmaphysik

Teilinstitut Greifswald

Wendelsteinstraße 1

17491 Greifswald

Germany

Tel: +49 - (0) 3834 / 882717

E-Mail: joris.fellinger@ipp.mpg.de 


\title{
Dynamic response analysis of superconducting coils in Wendelstein 7-X and mechanical quench test
}

\author{
Joris Fellinger, S. Freundt, D. Hathiramani, V. Bykov and F. Schauer \\ ${ }^{a}$ Max-Planck-Institut für Plasmaphysik, EURATOM Association, Teilinstitut Greifswald, \\ Wendelsteinstraße 1, D-17491 Greifswald, Germany
}

\begin{abstract}
The Wendelstein 7-X (W7-X) modular stellarator is under construction at the Max-Planck-Institut für Plasmaphysik in Greifswald, Germany. The W7-X magnet system contains 70 superconducting (sc) coils which are supported by a massive central steel ring structure via bolted connections and by numerous welded as well as gliding inter-coil elements.

Load tests on prototypes of bolted coil to support ring connections whose flanges partially open during high field operation, and of sliding support structures between the coils showed that stick-slip events at their contact surfaces cause shock waves. In order to assess the risk of a shock-induced quench of the superconductor, one of the coils was subjected to impact loads - the so called mechanical quench (MQ) test - in the course of the cryogenic acceptance tests at CEA in Saclay, France. No quench could be triggered even with the lowest coil stability margin.

The paper demonstrates that the amplitudes of the shock waves in the W7-X magnet system are comparable to those generated by the impacts in the MQ tests. For that purpose, finite element (FE) models of both W7-X and the MQ test simulating the dynamic responses were developed and successfully validated against measurements.
\end{abstract}

Keywords: Superconducting coil, mechanical disturbance, mechanical quench, dynamic response, FEM, W7-X

\section{Introduction}

The sc magnet system of the W7-X stellarator experiment consists of 50 non-planar and 20 planar coils which are supported by the central support structure and inter-coil structure elements [1]. This highly loaded support is prone to mechanical disturbances like stickslip effects at bolted central support elements (CSEs) with partly opening flanges and gliding narrow support elements (NSEs), as determined in tests, even though the CSE wedges (not the shims) and the NSE surfaces are covered with $\mathrm{MoS}_{2}$ as lubricant [2-4]. On the other hand, the coils are built up from cable-in-conduitconductors (CICC). In small scale tests on the sc cable compacted by compressive force in the transverse direction (to mimic the Lorenz force), the loadcompaction diagram showed hysteresis indicating that some of the energy stored in the cable during coil activation is converted into frictional heat upon compaction of the cable [5]. Moreover, these tests showed that shock waves could induce further frictional strand re-arrangements. The generated heat might come into the order of the conductor stability limit and possibly cause a quench. Notably, the stability margin of the CICC in W7-X is rather small as compared to other large sc devices [6]. Therefore, a series of MQ tests on a non-planar coil was performed under cryogenic conditions to evaluate the quench stability of the coil for various stability margins against mechanical impact [7].

The objective of the paper is to demonstrate that the dynamic response of the coil in the MQ tests is comparable to the response that must be expected in W7-X within the frequency domain which is relevant for triggering possible sc strand movements.
Therefore, modal analysis and transient dynamic analysis were carried out with FE models of the coil using Abaqus and Ansys FE software. In section two the relevant frequency spectrum is determined based on the one hand on the expected spectrum generated by stickslip events at the bolted CSEs and NSEs and on the other hand on the eigenfrequencies of the sc strands. In section three the displacement and acceleration measurements are compared in the frequency domain to prove the consistency of the measured data. The eigenfrequencies of the modal analysis were matched to the measured spectral distributions, and the damping required for the transient dynamic simulations was calibrated on the basis of the observed dispersion. In section four the transient dynamic FE models are validated by comparison of the projected dynamic response of the MQ tests with the measurements in the frequency domain. They are also compared with the projected dynamic response to stick-slip events at the CSEs and NSEs in W7-X to demonstrate that the mechanical disturbance in the MQ test is representative for W7-X.

\section{Relevant frequency spectrum}

\subsection{Generated frequencies}

Stick-slip events at CSE or NSE contacts due to the drop of the friction coefficient results in a sudden release of shear force. The frequency spectrum of the generated shock waves depends strongly on the release time of the shear forces. According to [8], it can be assumed that the shear force is released by a shear crack propagating over the contact, so the release time can be estimated based on the maximum crack front speed. According to [9], the crack front propagates over the contact area with the Rayleigh velocity of surface waves which is in the order 
of the transverse wave speed. The transverse speed is approximately 3100 and $2500 \mathrm{~m} / \mathrm{s}$ for steel and alubronze of the NSE pads respectively with the lubricant to be assumed as weared off. So the release of the shear force on a CSE contact with a typical size of $0.18 \mathrm{~m}$ requires some $60 \mu \mathrm{s}$. On a highly loaded NSE pad with a contact Hertz radius of some $40 \mathrm{~mm}$, the release time $t_{\text {rel }}$ is about $13 \mu \mathrm{s}$, conservatively calculated with the wave speed of steel.

The maximal frequency to be expected can be estimated by assuming a cosine shaped function for release of the shear force. Frequencies are then generated up to roughly $f_{u}=2 / t_{r e l}$, i.e. for a release time of $13 \mu \mathrm{s}$ the highest frequency is $150 \mathrm{kHz}$.

\subsection{Relevant amplitudes and frequencies}

Frictional heat is proportional to the sliding amplitude between the wires of the sc cable. Sliding is initiated by the change of contact stresses of the mostly elastically pre-stressed strands due to the inertia forces caused by the shock waves. This way avalanche like strand movements might be triggered which could release enough frictional heat beyond the stability margin of the cable $[5,7]$. However, any realistic friction event, including stick-slip, is associated with minimal relative movements on the order of several $\mu \mathrm{m}$ which is the size of the surface asperities of the contact $[10,11]$. Therefore, sliding amplitudes smaller than say $1 \mu \mathrm{m}$ are considered irrelevant.

The sliding amplitude results from Lorentz force, the stored elastic wire energy and support distances, but also from the amplitude of the shock waves, amplified by resonance of the wires.

Resonance of the wires increases the change in the contact stresses. Assuming a transverse wave is passing through a simply supported beam with span $l$, the $n^{\text {th }}$ eigenfrequency $f_{n}$ can be calculated with Young's modulus $E$, second moment of inertia $I$, density $\rho$ and cross sectional area $A$ as [12]

$$
f_{n}=\frac{\omega_{n}}{2 \pi}=\frac{1}{2 \pi} \frac{n^{2} \pi^{2}}{l^{2}} \sqrt{\frac{E I}{\rho A}}
$$

The wires in $\mathrm{W} 7-\mathrm{X}$ have $0.57 \mathrm{~mm}$ diameter and are arranged according to the cabling law of $3 \times 3 \times 3 \times 3 \times 3$. The span of the wires between the contact points is estimated from photos and scans to be in the order of several $\mathrm{mm}$ and proportional to the pitch length. The lowest eigenfrequency for single and bundled wires are calculated based on an upper bound of $l=1 / 3 l_{\text {pitch }}$, which results in a span of $8 \mathrm{~mm}$ for the single wire, a Young's modulus and density of copper of $120 \mathrm{GPa}$ and $8940 \mathrm{~kg} / \mathrm{m}^{3}$, resp., and estimated cross sectional properties. Those properties are based on the simple bundle composition of figure 1 in which $d_{i}, A_{i}$ and $I_{i}$ are the diameter, cross sectional area and moment of inertia, resp., of bundle $i$, and $\alpha_{i}$ a correction factor to account for the compaction of the wires relative to the principle of figure 1. With $\alpha_{1}=1$ and $\alpha_{i}=0.8$ for $i>1$, the diameter of the $3 \times 3 \times 3 \times 3 \times 3$ cable is $10.8 \mathrm{~mm}$ which matches the actual cable diameter reasonably well.

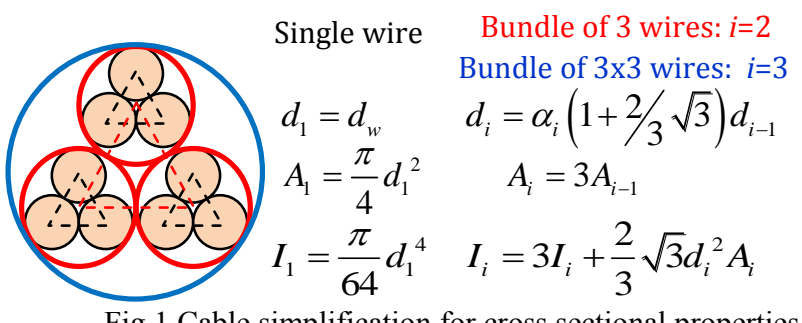

Fig.1 Cable simplification for cross sectional properties

The first eigenfrequency is $12 \mathrm{kHz}$ for the single wire and 5 to $6 \mathrm{kHz}$ for the bundles of wires. Forced vibration of the wires causes an excitation magnitude (without damping) that depends on the frequencies as [12]

$$
u_{\text {mag }} \propto \frac{1}{n^{4}} \frac{1}{1-\left(\omega / \omega_{n}\right)^{2}} .
$$

Eq. (3) clearly shows that second and higher eigenfrequencies are hardly resonating. Moreover, higher frequencies damp out more quickly. Therefore, the frequency domain is estimated to be relevant up to the highest first eigenfrequency, i.e. $12 \mathrm{kHz}$.

\subsection{Model requirements}

In order to resolve the shortest waves with wave length $\lambda_{u}$ with at least two elements, the mesh size $h_{c r}$ of the FE model must be limited to

$$
h_{c r} \leq \frac{1}{2} \lambda_{u}=\frac{1}{2} \frac{c_{2}}{f_{u}}=\frac{1}{2} \sqrt{\frac{G}{\rho}} \frac{1}{f_{u}}
$$

in which $G$ is the shear modulus of the solid medium and $c_{2}$ the transversal wave speed. For steel and aluminium, this leads to $h_{c r} \leq 130 \mathrm{~mm}$. For the FE models, coil meshes are extracted from the global models [13]. The actual mesh of the coils contains elements of typically $80 \times 40 \times 40 \mathrm{~mm}$ size, so the highest frequency waves can be well described. For the same reason the time increment in the implicit dynamic models must be limited to $d t \leq 1 /\left(2 f_{u}\right)=40 \mu s$. In explicit dynamic models the time increment is further limited by the mesh and automatically adjusted by the software.

\section{Validation of the model}

\subsection{Test results}

In addition to the MQ tests with various stability margins, two impact tests were carried out without coil current. These tests used an impact of a $10 \mathrm{~kg}$ pendulum mass fallen from $2 / 3 h$ with $h$ the maximum height of pendulum of $1.24 \mathrm{~m}$ (indicated as $\mathrm{N} 10 \mathrm{~m} 23 \mathrm{~h}$ ), and $30 \mathrm{~kg}$ mass fallen from $1 / 3 h(\mathrm{~N} 30 \mathrm{~m} 13 \mathrm{~h})$ [7]. Acceleration was measured using accelerometers, relative displacements along the diameter of the coil using strain gauges on cantilevered beams, and the impact force using strain gauges on the transfer rod [14], see figure 2. 
The strain gauge measurements in the tests with coil current are biased by inductive currents generated by movements of the sensor in the magnetic field. Therefore, the validation of the models concentrates on tests without current. The acceleration sensors are calibrated up to $5 \mathrm{kHz}$. They are not biased by the current and it was found that they show good agreement between tests with and without current.

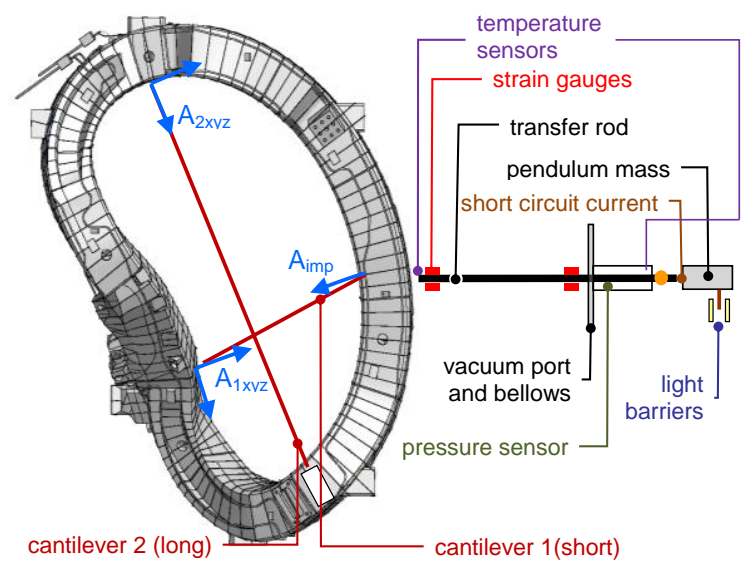

Fig. 2 Overview of MQ test set up.

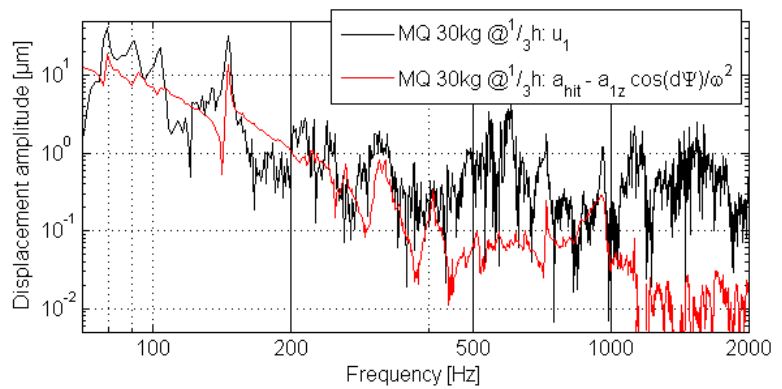

Fig. 3 Comparison of based on displacements and accelerations in MQ tests without current.

In figure 3, the amplitude spectral densities (ASD) of displacement $u_{1}$ of cantilever 1 are compared with the displacements derived from accelerations $a_{i m p}$ and $a_{1 z}$ (the z-direction is closest to the cantilever direction). The ASD was calculated using discrete Fourier transformation with zero padding. The sampling rate is $100 \mathrm{kHz}$ and the time window is from $0-500 \mathrm{~ms}$ relative to the time point where the pendulum mass hits the transfer rod. The raw displacement data were first shifted to obtain a zero mean over the entire measurement domain of $1.3 \mathrm{~s}$, i.e. the cool down displacement was subtracted. The mean of each raw acceleration measurement is also zero. For each sine-shaped component of the transformed displacement, the acceleration amplitude $\hat{u}_{n}$ is proportional to $\hat{a}_{n} / \omega_{n}^{2}$. For the acceleration difference between both ends of cantilever 1, the phase shift must be considered. Since the acceleration at the impact is an order of magnitude higher than at the opposite site, the acceleration difference is in phase with the impact acceleration, i.e. $d \hat{a}=\left(\hat{a}_{i m p, \omega}-\hat{a}_{1 z, \omega} \cos d \psi\right)$ with the phase shift $d \psi \approx 0$.

No significant amplitude beyond $2000 \mathrm{~Hz}$ was measured. In both tests both measurement types clearly show a peak at $147 \mathrm{~Hz}$. So the measurements are con- sistent, at least for low frequencies. At higher frequencies, the differences exist but these are not so relevant since the displacement magnitude is small $(<<1 \mu \mathrm{m})$.

The measured displacements are used to determine the damping ratio based on the dispersion near the peak amplitudes as $\zeta_{f}=1 / 2 \Delta f / f$, in which $\Delta f$ is the width of peak at frequency $f$ in the ASD measured at the level of $\hat{u}_{\text {peak }} / \sqrt{2}$. For both impact tests without coil current the damping ratio was calculated using the cantilever data at specific frequencies between $147-2049 \mathrm{~Hz}$, leading to damping ratios of $0.1-1.0 \%$. These frequencies were carefully selected to ensure that the dispersion was not biased by adjacent eigenfrequencies.

\subsection{Modal analysis}

The eigenfrequencies of the coil were determined with modal analyses in Ansys and Abaqus. For that purpose, the FE model of the coil was extracted from the W7-X global model [13] and a simple beam model for the support system in the test bed was added. In the test, the coil was suspended by three slender rods bolted to mounting blocks. Both FE models use linear solid elements with hourglass control. The winding pack (WP) and ground isolation are modelled with smeared orthotropic properties without details of the sc cables. The contact surfaces between the embedding and the coil case are simply tied.

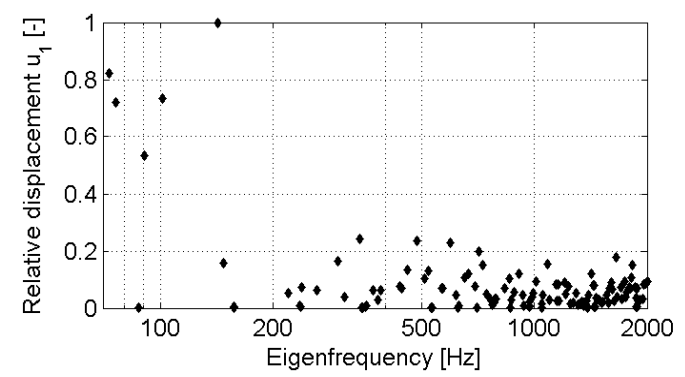

Fig. $4 \hat{u}_{1}$ based on modal analysis in Abaqus.

The lowest eigenfrequencies represent rigid body movements of the coil like a pendulum. The first relevant eigenmode of the coil exists at about $70-90 \mathrm{~Hz}$, slightly depending on the assumed stiffness of the support connection. Effects of gravity and coil current on the eigenmodes are negligible. In figure $4, \hat{u}_{1}$ for each eigenmode is shown. The maximum at $142 \mathrm{~Hz}$ agrees well with the observed peak at $147 \mathrm{~Hz}$ in figure 3 .

\subsection{Dynamic response of the MQ test}

The dynamic response in the MQ test set up was simulated for both tests without coil current using Abaqus 6.10 [15]. Rayleigh damping is applied given by

$$
\zeta_{\omega}=\frac{\alpha_{R}}{2 \omega}+\frac{\beta_{R} \omega}{2}
$$

$\alpha_{R}=18.6 \mathrm{rad} / \mathrm{s}$ and $\beta_{R}=6.0 \cdot 10^{-8} \mathrm{~s} / \mathrm{rad}$ are the mass and stiffness proportional damping factors respectively, fitted on the three measured damping ratios using the method of least squares. In addition, a default $5 \%$ 
numerical damping was applied to improve the numerical stability which damps out high frequency noise but does not significantly affect the results. The transfer rod was not modelled in detail. Instead the impact force history measured on the rod near the coil was smeared out as load on four nodes closest to the impact location.

In figure 5 , the ASD of $\hat{u}_{1}$ based on the accelerations are compared between simulation and test with the $30 \mathrm{~kg}$ mass dropped at $1 / 3$ of the maximum pendulum height. It shows that the projected ASD corresponds reasonably well with the measured one. Therefore, it is concluded that the FE model is capable of projecting the dynamic response of the coils.

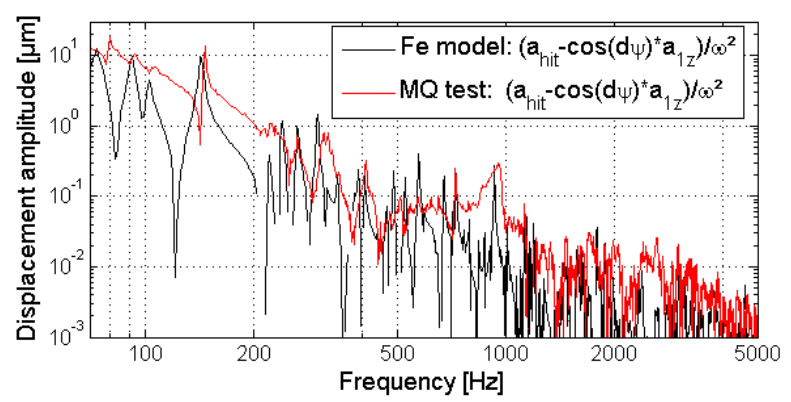

Fig. 5 ASD comparison between test and model.

\section{Comparison between MQ test and W7-X}

Next step was the comparison of the maximum accelerations along the inner edges of the (WP) where the highest magnetic field occurs. Those obtained in the MQ test are compared with those to be expected by credible worst case stick-slip scenarios at CSEs and NSEs in W7-X.

The highest impact in the MQ test was a drop of a $30 \mathrm{~kg}$ mass at $2 / 3$ of the maximum pendulum height. As force measurements in this MQ test were biased by the current, the force history measured in a preliminary test of the transfer rod against a wall was used as input in the FE model. For the same impact, force measurements obtained in this test set up are comparable to those measured in the MQ test without current.

The worst case NSE scenario is a drop of the friction of the highest loaded NSE pad from 0.1 to 0.05 in $13 \mu \mathrm{s}$, which is an upper bound compared to measured drops in tests [16]. The low friction of only 0.1 is due to the coating on the pads. Although shock waves generated at this NSE could cause stick-slip events in adjacent NSEs, a simultaneous release of the shear force at multiple NSEs is not considered, since there will be a time delay between the generated shocks.

Two CSE scenario's were considered: a drop of the friction coefficient in $60 \mu \mathrm{s}$ at the shim of NPC1Z1 from 0.5 to 0.35 and from 0.35 to 0.3 which are the extremes found over multiple cool down cycles in steel on steel friction tests at cryogenic conditions [17] and a drop from 0.1 to 0.05 at the $\mathrm{MoS}_{2}$-coated wedges.

In figure 6 , the ASD of the maximum accelerations anywhere along the edges of the WP are divided by $\omega_{n}^{2}$.
As the figure shows, the amplitudes in the MQ test are at least equal to those expected in the worst case scenario for W7-X over the entire frequency domain.

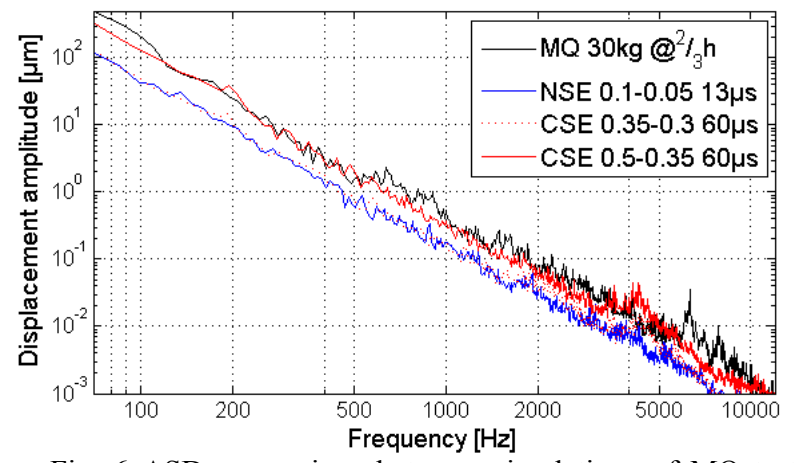

Fig. 6 ASD comparison between simulations of MQ test and stick slip events in W7-X

\section{Conclusions}

The dynamic response of the superconducting W7-X coil to the impact exerted in the MQ test was successfully simulated using FE models within the relevant frequency domain of $0.07-12 \mathrm{kHz}$.

The comparison of the dynamic WP responses between the MQ test and credible worst case impact loads in W7-X demonstrates that the impact in the MQ test is representative for $\mathrm{W} 7-\mathrm{X}$. Since no coil quenches occurred in the MQ tests even for the smallest stability margin, no quenches due to stick slip events within the W7-X structure have to be expected.

\section{Acknowledgments}

The contributions of $\mathrm{H}$. Langer, C. Zauner and Pfeiffer of KRP Mechatec, Germany, of G. Samuelli and F. Lucca of L.T. Calcoli, Italy and of Th. Bergmann of IPP Greifswald are greatly acknowledged.

\section{References}

[1] L. Wegener et al., Fus. Eng. Des. 84, pp.106-112 (2009)

[2] A. Dudek et al., Fus. Eng. Des. 84, pp. 703-706 (2009)

[3] D. Hathiramani et al., Fus.Eng.Des.84, pp. 899-902 (2009)

[4] C. Zauner et al., Fus. Eng. Des. 84, pp. 2042-2045 (2009)

[5] F. Schauer, IEEE Tr. on Magn. 32, 4, pp. 2760-2763 (1996)

[6] K. Seo et al., Fus. Eng. Des. 81, pp. 2497-2502 (2006)

[7] D. Hathiramani et al., IEEE Tr. on Appl. Superc. 20, pp. 543-546 (2010).

[8] E. Brener and V. Marchenko, JETP Letters, 76, 4, pp. 211-214 (2002)

[9] L.B. Freund, Dynamic fracture mechanics, Cambridge University Press, 1994)

[10] D. Tabor, Proc. Royal Soc. London, A, 251, 1266 pp. 378-393 (1959)

[11] E. Rabinowitz, Proc. Phys. Soc. 71, 4, pp. 668-675 (1958)

[12] S. Timoshenko, Vibration problems in engineering, (Wiley \& Sons, NY, 1974) p. 421

[13] V. Bykov et al., Fus. Eng. Des. 84, pp.215-219 (2009)

[14] J.P. Kallmeyer et al., Proc. SOFT 2010 (this conference)

[15] Abaqus Documentation version 6.10 (2010)

[16] Th. Gradt et al. Fus. Eng. Des. 84, pp. 840-843 (2009)

[17] J.C. Burton et al., Tribo. Letters 23,2, pp. 131-137 (2006) 